\title{
Existence of Periodic Solutions for Shunting Inhibitory Cellular Neural Networks with Neutral Delays
}

\author{
Ninghua Chen \\ Fujian Preschool Education College, Fuzhou, Fujian 350007, China \\ Correspondence should be addressed to Ninghua Chen; cnh166@sohu.com
}

Received 3 July 2013; Accepted 16 September 2013

Academic Editor: Cecile Piret

Copyright ( 2013 Ninghua Chen. This is an open access article distributed under the Creative Commons Attribution License, which permits unrestricted use, distribution, and reproduction in any medium, provided the original work is properly cited.

This paper considers the existence of periodic solutions for shunting inhibitory cellular neural networks (SICNNs) with neutral delays. By applying the theory of abstract continuation theorem of $k$-set contractive operator and some analysis technique, a new result on the existence of periodic solutions is obtained.

\section{Introduction}

It is well known that SICNNs have been extensively applied in psychophysics, speech, perception, robotics, adaptive pattern recognition, vision, and image processing. The dynamical behaviors of SICNNs with delays have been widely investigated in recent years. A large number of important results on the dynamical behaviors of SICNNs have been established and successfully applied to signal processing, pattern recognition, associative memories, and so on. In particular, there exist many results on the existence and stability of periodic and almost periodic solutions for SICNNs with delays. We refer the reader to [1-4] and references cited therein.

On the other hand, the stability analysis of various neutral delay-differential systems has drawn much research attention [5-7]. The theory of neutral delay-differential systems is of both theoretical and practical interest. For a large class of electrical networks containing lossless transmission lines, the describing equations can be reduced to neutral delaydifferential equations; such networks arise in high speed computers where nearly lossless transmission lines are used to interconnect switching circuits. Also, the neutral systems often appear in the study of automatic control, population dynamics, and vibrating masses attached to an elastic bar.
Motivated by the above, we consider shunting inhibitory cellular neural networks with neutral delays described by

$$
\begin{aligned}
\dot{x}_{i j}(t)= & -a_{i j}(t) x_{i j}(t) \\
& -\sum_{C_{k l} \in N_{r}(i, j)} C_{i j}^{k l}(t) f_{i j}\left(x_{k l}\left(t-\tau_{k l}(t)\right)\right) x_{i j}(t) \\
& +b_{i j}(t) \dot{x}_{i j}\left(t-\sigma_{i j}(t)\right)+I_{i j}(t),
\end{aligned}
$$

where $i=1,2, \ldots, m, j=1,2, \ldots, n, C_{i j}$ is the cell at the $(i, j)$ position of the lattice and the $r$-neighborhood $N_{r}(i, j)$ of $C_{i j}$ is given as

$$
\begin{aligned}
& N_{r}(i, j)=\left\{C_{k l}: \max (|k-i|,|l-j|)\right. \\
&\leq r, 1 \leq k \leq m, 1 \leq l \leq n\} .
\end{aligned}
$$

$x_{i j}(t)$ is the activity of the cell, $C_{i j}, a_{i j}(t) \geq 0$ is the passive decay rate of the cell activity, $I_{i j}(t)$ is the external input to $C_{i j}$, $C_{i j}^{k l} \geq 0$ is the connection or coupling strength of postsynaptic activity of the cell transmitted to the cell $C_{i j}$, and the activity function $f_{i j}\left(x_{k l}(t)\right)$ is a continuous function representing the output or firing rate of cell $C_{k l}$. 
By using the Lyapunov functions and linear matrix inequality approach (LMI), most authors studied the asymptotic stability or exponential stability of the equilibrium point (see [7-10]). However, few papers have been published on the existence of periodic solutions or almost periodic solutions for neutral type neural networks ([11-14]). Thus, it is worthwhile to study the existence of periodic solutions for neutral type neural networks with delays. And to the author's best knowledge, there is no published paper considering the existence of periodic solutions for SICNNs with neutral delays.

The initial conditions of (1) are of the form

$$
\begin{aligned}
x_{i j}(t) & =\varphi_{i j}(s), \quad s \in(-\infty, 0], \\
i & =1, \ldots, m, \quad j=1, \ldots, n,
\end{aligned}
$$

where $\varphi_{i j} \in C((-\infty, 0], R)$.

For the sake of convenience, we denote

$$
\begin{gathered}
\Omega_{1}=\{1,2, \ldots, m\}, \quad \Omega_{2}=\{1,2, \ldots, n\}, \\
\bar{h}=\max _{t \in[0, \omega]}|h(t)|, \quad \underline{h}=\min _{t \in[0, \omega]}|h(t)|, \\
\widetilde{h}=\frac{1}{\omega} \int_{0}^{\omega} h(s) d s, \\
\|h\|_{2}=\left(\int_{0}^{\omega}|h(t)|^{2} d t\right)^{1 / 2}, \quad\|\dot{h}\|_{2}=\left(\int_{0}^{\omega}|\dot{h}(t)|^{2} d t\right)^{1 / 2},
\end{gathered}
$$

where $h$ is a continuous $\omega$-periodic function.

The main purpose of this paper is to obtain some sufficient conditions for the existence of the periodic solutions for system (1). By applying the theory of abstract continuation theorem of $k$-set contractive operator and some analysis technique, we derive some new sufficient conditions ensuring the existence of periodic solutions for system (1). Moreover, numerical examples are provided to illustrate the feasibility of the new results.

\section{Conditions and Lemma}

Throughout this paper, for $i \in \Omega_{1}, j \in \Omega_{2}$, we assume that

$\left(\mathrm{H}_{1}\right) a_{i j}(t), C_{i j}^{k l}(t), b_{i j}(t), I_{i j}(t), \tau_{i j}(t)$ are continuous $\omega$ periodic functions;

$\left(\mathrm{H}_{2}\right)$ function $\sigma_{i j}(t)$ is nonnegative, bounded, $\omega$-periodic, and continuously differential defined on $R^{+}$and $\inf _{t \in R^{+}}\left\{1-\dot{\sigma}_{i j}(t)\right\}>0$, where $\dot{\sigma}_{i j}(t)$ expresses the derivative of $\sigma_{i j}(t)$;

$\left(\mathrm{H}_{3}\right)$ there exists positive constant $\alpha_{i j}$ such that $\left|f_{i j}(u)\right| \leq$ $\alpha_{i j}$, for all $u, v \in R$.

In order to study (1), we should make some preparations.
Let $E$ be a Banach space. For a bounded subset $A \subset E$, let

$\alpha_{E}(A)$

$$
\begin{aligned}
& =\inf \{\delta>0: \text { there is a finite number of subsets } \\
& \left.\qquad A_{i} \subset A \text { such that } A=\bigcup_{i} A_{i} \text { and } \operatorname{diam}\left(A_{i}\right) \leq \delta\right\}
\end{aligned}
$$

denote the (Kuratowski) measure of noncompactness, where diam $\left(A_{i}\right)$ denotes the diameter of the set $A_{i}$. Let $X, Y$ be two Banach spaces and $\Omega$ be a bounded open subset of $X$. A continuous and bounded map $N: \bar{\Omega} \rightarrow Y$ is called $k$-set contractive if for any bounded set $S \subset \Omega$ we have $\alpha_{Y}(N(S)) \leq k \alpha_{X}(S)$, where $k$ is a constant. In addition, for a Fredholm operator $L: X \rightarrow Y$ with index zero, according to [15], we define

$$
\begin{array}{r}
l(L)=\sup \left\{a \geq 0: a \alpha_{X}(S) \leq \alpha_{Y}(L(S)),\right. \\
\forall \text { bounded subset } S \subset X\} .
\end{array}
$$

Lemma 1 (see [16]). Let $L: X \rightarrow Y$ be a Fredholm operator with index zero, and let $r \in Y$ be a fixed point. Suppose that $N: \Omega \rightarrow Y$ is a $k$-set contractive with $k<l(L)$, where $\Omega \subset X$ is bounded, open, and symmetric about $0 \in \Omega$. Furthermore, we suppose that

(1) $L x \neq \lambda N x+\lambda r$ for $x \in \partial \Omega, \lambda \in(0,1)$,

(2) $[Q N(x)+Q r, x] \cdot[Q N(-x)+Q r, x]<0$ for $x \in \partial \Omega \cap$ $\operatorname{Ker} L$, where $[\cdot, \cdot]$ is some bilinear form on $Y \times X$ and $Q$ is the project of $Y$ onto Coker $(L)$.

Then there is a $x \in \bar{\Omega}$ such that $L x-N x=r$.

In order to use Lemma 1 to establish the existence of periodic solutions of (1), we set

$$
\begin{array}{r}
Y=C_{\omega}=\{x \in C(R, R): x(t+\omega)=x(t), \\
x=\left(x_{11}, \ldots, x_{1 n}, \ldots, x_{i 1}, \ldots,\right. \\
\left.\left.x_{i n}, \ldots, x_{m 1}, \ldots, x_{m n}\right)^{T}\right\}
\end{array}
$$

with the norm defined by $\|x\|=\sum_{i=1}^{m} \sum_{j=1}^{n}\left|x_{i j}\right|_{0}$, where $\left|x_{i j}\right|_{0}=\max _{t \in[0, \omega]}\left|x_{i j}(t)\right|$ and

$$
\begin{aligned}
& X= C_{\omega}^{1} \\
&=\left\{x \in C^{1}(R, R): x(t+\omega)=x(t),\right. \\
& x=\left(x_{11}, \ldots, x_{1 n}, \ldots, x_{i 1}, \ldots,\right. \\
&\left.\left.\quad x_{i n}, \ldots, x_{m 1}, \ldots, x_{m n}\right)^{T}\right\}
\end{aligned}
$$


with the norm defined by $\|x\|_{1}=\max \{\|x\|,\|\dot{x}\|\}$, where $\|\dot{x}\|=\sum_{i=1}^{m} \sum_{j=1}^{n}\left|\dot{x}_{i j}\right|_{0},\left|\dot{x}_{i j}\right|_{0}=\max _{t \in[0, \omega]}\left|\dot{x}_{i j}(t)\right|$. Then $X, Y$ are Banach spaces.

Let $L: X \rightarrow Y$ defined by

$$
\begin{array}{r}
L x=\frac{d x}{d t}=\left(\dot{x}_{11}, \ldots, \dot{x}_{1 n}, \ldots, \dot{x}_{i 1}, \ldots,\right. \\
\left.\dot{x}_{i n}, \ldots, \dot{x}_{m 1}, \ldots, \dot{x}_{m n}\right)^{T}
\end{array}
$$

and $N: X \rightarrow Y$ defined by the following:

$$
\begin{aligned}
N x_{i j}= & -a_{i j}(t) x_{i j}(t) \\
& -\sum_{C_{k l} \in N_{r}(i, j)} C_{i j}^{k l}(t) f_{i j}\left(x_{k l}\left(t-\tau_{k l}(t)\right)\right) x_{i j}(t) \\
& +b_{i j}(t) \dot{x}_{i j}\left(t-\sigma_{i j}(t)\right),
\end{aligned}
$$

for $i \in \Omega_{1}, j \in \Omega_{2}$.

It is easy to see that $L$ is a Fredholm operator with index zero. Clearly, (1) has a $\omega$-periodic solution if and only if $L x=$ $N x+r$ for some $x \in X$, where

$$
\begin{aligned}
r:=I(t)= & \left(I_{11}(t), \ldots, I_{1 n}(t), \ldots, I_{i 1}(t), \ldots,\right. \\
& \left.I_{i n}(t), \ldots, I_{m 1}(t), \ldots, I_{m n}(t)\right)^{T} .
\end{aligned}
$$

Lemma 2 (see [17]). The differential operator $L$ is a Fredholm operator with index zero and satisfies $l(L) \geq 1$.

Lemma 3. If $k=\max _{i \in \Omega_{1}, j \in \Omega_{\Omega}}\left\{\bar{b}_{i j}\right\}<1$, then $N: \bar{\Omega} \rightarrow Y$ is a $k$-set contractive map, where $\Omega \subset X$.

Proof. The proof of Lemma 3 is similar to Lemma 3 in [14] and will be omitted here.

Lemma 4 (see [13]). Let $t_{1}, t_{2} \in[0, \omega]$. If $x:[0, \omega] \rightarrow R$ is $\omega$-periodic, then

$$
\begin{aligned}
& x(t) \leq x\left(t_{1}\right)+\int_{0}^{\omega}|\dot{x}(s)| d s, \\
& x(t) \geq x\left(t_{2}\right)-\int_{0}^{\omega}|\dot{x}(s)| d s .
\end{aligned}
$$

Lemma 5 (see [18]). Suppose that $\sigma \in X$ and $\dot{\sigma}(t)<1$ for all $t \in[0, \omega]$, then the function $t-\sigma(t)$ has an inverse $v \in C(R, R)$ with $\nu(a+\omega)=\nu(a)+\omega$, for all $a \in R$.

Throughout this paper, we assume that $\sigma_{i j} \in X$ and $\dot{\sigma}_{i j}(t)<1, i \in \Omega_{1}, j \in \Omega_{2}$. So that $\sigma_{i j}(t)$ has a unique inverse, and we set $v_{i j}(t)$ to represent the inverse of function $t-\sigma_{i j}(t)$.

\section{Main Results}

Set

$$
\begin{gathered}
p_{i j}^{2}=\max _{t \in R}\left\{\frac{1}{1-\dot{\sigma}_{i j}\left(v_{i j}(t)\right)}\right\}, \\
A=\operatorname{diag}\left(\underline{a}_{11}, \ldots, \underline{a}_{1 n}, \ldots, \underline{a}_{m 1}, \ldots, \underline{a}_{m n}\right), \\
B=\operatorname{diag}\left(\bar{b}_{11} p_{11}, \ldots, \bar{b}_{1 n} p_{1 n}, \ldots, \bar{b}_{m 1} p_{m 1}, \ldots, \bar{b}_{m n} p_{m n}\right), \\
D=\operatorname{diag}\left(1-\bar{b}_{11} p_{11}, \ldots, 1-\bar{b}_{1 n} p_{1 n}, \ldots,\right. \\
\left.1-\bar{b}_{m 1} p_{m 1}, \ldots, 1-\bar{b}_{m n} p_{m n}\right), \\
C=\operatorname{diag}\left(\sum_{C_{k l} \in N_{r}(i, j)} \bar{C}_{11}^{k l} \alpha_{11}, \ldots, \sum_{C_{k l} \in N_{r}(i, j)} \bar{C}_{1 n}^{k l} \alpha_{1 n}, \ldots,\right. \\
\left.\sum_{C_{k l} \in N_{r}(i, j)} \bar{C}_{m 1}^{k l} \alpha_{m 1}, \ldots, \sum_{C_{k l} \in N_{r}(i, j)} \bar{C}_{m n}^{k l} \alpha_{m n}\right), \\
Z=\left(\left\|I_{11}\right\|_{2}, \ldots,\left\|I_{1 n}\right\|_{2}, \ldots,\left\|I_{m 1}\right\|_{2}, \ldots,\left\|I_{m n}\right\|_{2}\right)^{T}, \\
W=\left(\left\|x_{11}\right\|_{2}, \ldots,\left\|x_{1 n}\right\|_{2}, \ldots,\left\|x_{m 1}\right\|_{2}, \ldots,\left\|x_{m n}\right\|_{2}\right)^{T},
\end{gathered}
$$

Theorem 6. Suppose that $\left(H_{1}\right)-\left(H_{3}\right)$ hold, furthermore, assume that

$$
\begin{aligned}
& \left(\mathrm{H}_{4}\right) \min _{i \in \Omega_{1}, j \in \Omega_{2}}\left\{1-\bar{b}_{i j} p_{i j}, 1-\bar{b}_{i j}\right\}>0 \text { and } \\
& \max _{i \in \Omega_{1}, j \in \Omega_{2}}\left\{\widetilde{a}_{i j}-\sum_{C_{k l} \in N_{r}(i, j)} \widetilde{C}_{i j}^{k l} \alpha_{i j}\right\}>0, \\
& \left(\mathrm{H}_{5}\right) \text { A }-C-B D^{-1}(A+C) \text { is nonsingular M-matrix, then } \\
& \text { system (1) has at least one } \omega \text {-periodic solution. }
\end{aligned}
$$

Proof. We consider the operator equation $L x=\lambda N x+\lambda r$, $\lambda \in(0,1)$, where $L, N$ defined by (9) and (10), respectively. Corresponding to system (1), for $i \in \Omega_{1}, j \in \Omega_{2}$, we have

$$
\begin{aligned}
\dot{x}_{i j}(t)=\lambda[ & -a_{i j}(t) x_{i j}(t) \\
& -\sum_{C_{k l} \in N_{r}(i, j)} C_{i j}^{k l}(t) f_{i j}\left(x_{k l}\left(t-\tau_{k l}(t)\right)\right) x_{i j}(t) \\
& \left.+b_{i j}(t) \dot{x}_{i j}\left(t-\sigma_{i j}(t)\right)+I_{i j}(t)\right] .
\end{aligned}
$$

Suppose that $\left(x_{11}(t), \ldots, x_{1 n}(t), \ldots, x_{m 1}(t), \ldots, x_{m n}(t)\right)^{T}$ is a solution of system (14) for a parameter $\lambda \in(0,1)$. Multiplying 
both sides of system (14) by $x_{i j}(t)$ and integrating over $[0, \omega]$, which gives from $\left(\mathrm{H}_{3}\right)$ and Cauchy-Schwarz inequality, we obtain

$$
\begin{aligned}
& \underline{a}_{i j}\left\|x_{i j}\right\|_{2}^{2} \\
& \leq \int_{0}^{\omega} a_{i j}(t) x_{i j}^{2}(t) d t \\
& =\int_{0}^{\omega}\left[-\sum_{C_{k l} \in N_{r}(i, j)} C_{i j}^{k l}(t) f_{i j}\left(x_{k l}\left(t-\tau_{k l}(t)\right)\right) x_{i j}(t)\right. \\
& \left.\quad+b_{i j}(t) \dot{x}_{i j}\left(t-\sigma_{i j}(t)\right)+I_{i j}(t)\right] x_{i j}(t) d t \\
& \leq \sum_{k l} \bar{C}_{r}^{k l}(i, j) \\
& \quad+\bar{b}_{i j} \int_{0}^{\omega}\left|x_{i j}(t)\right|\left|\dot{x}_{i j}\left(t-\sigma_{i j}(t)\right)\right|_{0}^{\omega} x_{i j}^{2}(t) d t \\
& \quad+\int_{0}^{\omega}\left|x_{i j}(t)\right|\left|I_{i j}(t)\right| d \\
& \leq \sum_{C_{k l} \in N_{r}(i, j)} \bar{C}_{i j}^{k l} \alpha_{i j}\left\|x_{i j}\right\|_{2}^{2} \\
& \quad+\left.\bar{b}_{i j}\left\|x_{i j}\right\|\right|_{2}\left(\int_{0}^{\omega}\left|\dot{x}_{i j}\left(t-\sigma_{i j}(t)\right)\right|^{2} d t\right)^{1 / 2} \\
& \quad+\left(\int_{0}^{\omega}\left|I_{i j}(t)\right|^{2} d t\right)^{1 / 2}\left(\int_{0}^{\omega}\left|x_{i j}(t)\right|^{2} d t\right)^{1 / 2},
\end{aligned}
$$

and according to Lemma 5, we have

$$
\begin{gathered}
\left(\int_{0}^{\omega}\left|\dot{x}_{i j}\left(t-\sigma_{i j}(t)\right)\right|^{2} d t\right)^{1 / 2} \\
\quad=\left(\int_{0}^{\omega} \frac{1}{1-\dot{\sigma}_{i j}\left(v_{i j}(s)\right)}\left|\dot{x}_{i j}(s)\right|^{2} d s\right)^{1 / 2} \\
\leq p_{i j}\left\|\dot{x}_{i j}\right\|_{2}, \quad i \in \Omega_{1}, \quad j \in \Omega_{2} .
\end{gathered}
$$

That is,

$$
\begin{aligned}
\underline{a}_{i j}\left\|x_{i j}\right\|_{2} \leq & \sum_{C_{k l} \in N_{r}(i, j)} \bar{C}_{i j}^{k l} \alpha_{i j}\left\|x_{i j}\right\|_{2} \\
& +\bar{b}_{i j} p_{i j}\left\|\dot{x}_{i j}\right\|_{2}+\left\|I_{i j}\right\|_{2}, \quad i \in \Omega_{1}, \quad j \in \Omega_{2} .
\end{aligned}
$$

From the above inequality, we obtain

$$
\begin{array}{r}
\left(\underline{a}_{i j}-\sum_{C_{k l} \in N_{r}(i, j)} \bar{C}_{i j}^{k l} \alpha_{i j}\right)\left\|x_{i j}\right\|_{2} \leq \bar{b}_{i j} p_{i j}\left\|\dot{x}_{i j}\right\|_{2}+\left\|I_{i j}\right\|_{2}, \\
i \in \Omega_{1}, \quad j \in \Omega_{2},
\end{array}
$$

$$
(A-C) Z \leq B W+I \text {. }
$$

Multiplying both sides of (14) by $\dot{x}_{i j}(t)$ and integrating over $[0, \omega]$, for $i \in \Omega_{1}, j \in \Omega_{2}$, we have

$$
\begin{aligned}
\left\|\dot{x}_{i j}\right\|_{2}^{2} & \\
= & \lambda \int_{0}^{\omega} \dot{x}_{i j}(t)\left[-a_{i j}(t) x_{i j}(t)\right. \\
& -\sum_{C_{k l} \in N_{r}(i, j)} C_{i j}^{k l}(t) f_{i j}\left(x_{k l}\left(t-\tau_{k l}(t)\right)\right) \\
\times x_{i j}(t)+b_{i j}(t) \dot{x}_{i j} & \left.\times\left(t-\sigma_{i j}(t)\right)+I_{i j}(t)\right] d t \\
\leq & \left(\bar{a}_{i j}+\sum_{C_{k l} \in N_{r}(i, j)} \bar{C}_{i j}^{k l} \alpha_{i j}\right)\left(\int_{0}^{\omega}\left|\dot{x}_{i j}(t)\right|^{2} d t\right)^{1 / 2} \\
& \times\left(\int_{0}^{\omega}\left|x_{i j}(t)\right|^{2} d t\right)^{1 / 2}+\bar{b}_{i j}\left(\int_{0}^{\omega}\left|\dot{x}_{i j}(t)\right|^{2} d t\right)^{1 / 2} \\
& \times\left(\int_{0}^{\omega}\left|\dot{x}_{i j}\left(t-\sigma_{i j}(t)\right)\right|^{2} d t\right)^{1 / 2} \\
& +\left(\int_{0}^{\omega}\left|I_{i j}(t)\right|^{2} d t\right)^{1 / 2}\left(\int_{0}^{\omega}\left|\dot{x}_{i j}(t)\right|^{2} d t\right)^{1 / 2} \\
& \left(\bar{a}_{i j}+\sum_{i j} p_{i j}\left\|\dot{x}_{i j}\right\|_{2}^{2}+\left\|\dot{x}_{i j}\right\|\left\|_{2}\right\| I_{i j} \|\left.\right|_{2} ^{k l} \cdot \alpha_{i j}\right)\left\|x_{i j}\right\|\left\|_{2}\right\| \dot{x}_{i j} \|_{2}
\end{aligned}
$$

Thus

$$
\begin{aligned}
\left\|\dot{x}_{i j}\right\|_{2} \leq & \left(\bar{a}_{i j}+\sum_{C_{k l} \in N_{r}(i, j)} \bar{C}_{i j}^{k l} \alpha_{i j}\right)\left\|x_{i j}\right\|_{2} \\
& +\bar{b}_{i j} p_{i j}\left\|\dot{x}_{i j}\right\|_{2}+\left\|I_{i j}\right\|_{2},
\end{aligned}
$$

that is,

$$
\begin{aligned}
\left(1-\bar{b}_{i j} p_{i j}\right)\left\|\dot{x}_{i j}\right\|_{2} \leq & \left(\bar{a}_{i j}+\sum_{C_{k l} \in N_{r}(i, j)} \bar{C}_{i j}^{k l} \alpha_{i j}\right)\left\|x_{i j}\right\|_{2} \\
& +\left\|I_{i j}\right\|_{2},
\end{aligned}
$$


where $1-\bar{b}_{i j} p_{i j}>0, i \in \Omega_{1}, j \in \Omega_{2}$. Then we may rewrite (22) as

$$
\begin{aligned}
D W & \leq(A+C) Z+I \Longrightarrow W \\
& \leq D^{-1}(A+C) Z+D^{-1} I .
\end{aligned}
$$

Substituting (23) into (19), we get

$$
\begin{aligned}
(A-C) Z & \leq B\left(D^{-1}(A+C) Z+D^{-1} I\right)+I \\
& \Longrightarrow\left(A-C-B D^{-1}(A+C)\right) Z \\
& \leq B D^{-1} I+I .
\end{aligned}
$$

By $\left(\mathrm{H}_{5}\right)$, we have $Z \leq H^{+} \triangleq\left(h_{11}^{+}, \ldots, h_{1 n}^{+}, \ldots, h_{m 1}^{+}, \ldots, h_{m n}^{+}\right)^{T}$. That is,

$$
\left\|x_{i j}\right\|_{2} \leq h_{i j}^{+}, \quad i \in \Omega_{1}, \quad j \in \Omega_{2},
$$

where $H^{+}=\left(A-C-B D^{-1}(A+C)\right)^{-1}\left(B D^{-1} I+I\right)$. Substituting (25) into (23), we have $W \leq G^{+} \triangleq\left(g_{11}^{+}, \ldots, g_{1 n}^{+}, \ldots\right.$, $\left.g_{m 1}^{+}, \ldots, g_{m n}^{+}\right)^{T}$. That is,

$$
\left\|\dot{x}_{i j}\right\|_{2} \leq g_{i j}^{+}, \quad i \in \Omega_{1}, \quad j \in \Omega_{2},
$$

where $G^{+}=D^{-1}(A+C) H^{+}+D^{-1} I$.

Integrating both sides of (14), from 0 to $\omega$, we obtain

$$
\begin{aligned}
0= & -\int_{0}^{\omega} a_{i j}(t) x_{i j}(t) d t \\
& +\int_{0}^{\omega}\left[-\sum_{C_{k l} \in N_{r}(i, j)} C_{i j}^{k l}(t) f_{i j}\left(x_{k l}\left(t-\tau_{k l}(t)\right)\right) x_{i j}(t)\right. \\
& \left.+b_{i j}(t) \dot{x}_{i j}\left(t-\sigma_{i j}(t)\right)+I_{i j}(t)\right] d t .
\end{aligned}
$$

Using Cauchy-Schwarz inequality, we have

$$
\begin{aligned}
& \left|\int_{0}^{\omega} a_{i j}(t) x_{i j}(t) d t\right| \\
& \leq \sum_{C_{k l} \in N_{r}(i, j)} \int_{0}^{\omega} \bar{C}_{i j}^{k l} \alpha_{i j}\left|x_{i j}(t)\right| d t \\
& \quad+\int_{0}^{\omega} \bar{b}_{i j}\left|\dot{x}_{i j}\left(t-\sigma_{i j}(t)\right)\right| d t+\int_{0}^{\omega}\left|I_{i j}(t)\right| d t \\
& \leq \sum_{C_{k l} \in N_{r}(i, j)} \bar{C}_{i j}^{k l} \alpha_{i j}\left\|x_{i j}\right\|_{2} \sqrt{\omega} \\
& \quad+\bar{b}_{i j} p_{i j}\left\|\dot{x}_{i j}\right\|_{2} \sqrt{\omega}+\left\|I_{i j}\right\|_{2} \sqrt{\omega} .
\end{aligned}
$$

In view of (14), we have

$$
\begin{aligned}
\int_{0}^{\omega}\left|\dot{x}_{i j}(t)\right| d t \leq & \int_{0}^{\omega} \bar{a}_{i j}\left|x_{i j}(t)\right| d t \\
& +\sum_{C_{k l} \in N_{r}(i, j)} \int_{0}^{\omega} \bar{C}_{i j}^{k l} \alpha_{i j}\left|x_{i j}(t)\right| d t \\
& +\int_{0}^{\omega} \bar{b}_{i j}\left|\dot{x}_{i j}\left(t-\sigma_{i j}(t)\right)\right| d t+\int_{0}^{\omega}\left|I_{i j}(t)\right| d t \\
\leq & \bar{a}_{i j}\left\|x_{i j}\right\|_{2} \sqrt{\omega} \\
& +\sum_{C_{k l} \in N_{r}(i, j)} \bar{C}_{i j}^{k l} \alpha_{i j}\left\|x_{i j}\right\|_{2} \sqrt{\omega} \\
& +\bar{b}_{i j} p_{i j}\left\|\dot{x}_{i j}\right\|_{2} \sqrt{\omega}+\left\|I_{i j}\right\|_{2} \sqrt{\omega} .
\end{aligned}
$$

From Lemma 4, for any $t_{1}^{i j}, t_{2}^{i j} \in[0, \omega], i \in \Omega_{1}, j \in \Omega_{2}$, we have

$$
\begin{aligned}
\int_{0}^{\omega} a_{i j}(t) x_{i j}(t) d t \leq & \int_{0}^{\omega} a_{i j}(t) x_{i j}\left(t_{1}^{i j}\right) d t \\
& +\int_{0}^{\omega} a_{i j}(t)\left(\int_{0}^{\omega}\left|\dot{x}_{i j}(t)\right| d t\right) d t, \\
\int_{0}^{\omega} a_{i j}(t) x_{i j}(t) d t \geq & \int_{0}^{\omega} a_{i j}(t) x_{i j}\left(t_{2}^{i j}\right) d t \\
& -\int_{0}^{\omega} a_{i j}(t)\left(\int_{0}^{\omega}\left|\dot{x}_{i j}(t)\right| d t\right) d t .
\end{aligned}
$$

Dividing by $\int_{0}^{\omega} a_{i j}(t) d t$ on the two sides of the above inequalities, we obtain that

$$
\begin{aligned}
& x_{i j}\left(t_{1}^{i j}\right) \geq \frac{1}{\int_{0}^{\omega} a_{i j}(t) d t} \int_{0}^{\omega} a_{i j}(t) x_{i j}(t) d t-\int_{0}^{\omega}\left|\dot{x}_{i j}(t)\right| d t, \\
& x_{i j}\left(t_{2}^{i j}\right) \leq \frac{1}{\int_{0}^{\omega} a_{i j}(t) d t} \int_{0}^{\omega} a_{i j}(t) x_{i j}(t) d t+\int_{0}^{\omega}\left|\dot{x}_{i j}(t)\right| d t .
\end{aligned}
$$

Let $\bar{t}_{i j}, \underline{t}_{i j} \in[0, \omega], i \in \Omega_{1}, j \in \Omega_{2}$, such that $x_{i j}\left(\bar{t}_{i j}\right)=$ $\max _{t \in[0, \omega]} x_{i j}(t), x_{i j}\left(\underline{t}_{i j}\right)=\min _{t \in[0, \omega]} x_{i j}(t)$, then by $(25)-(31)$, we have

$$
\begin{aligned}
x_{i j}\left(\bar{t}_{i j}\right) \leq & \frac{1}{\underline{a}_{i j} \omega} \int_{0}^{\omega}\left|a_{i j}(t) x_{i j}(t)\right| d t+\int_{0}^{\omega}\left|\dot{x}_{i j}(t)\right| d t \\
\leq & \frac{1}{\underline{a}_{i j} \sqrt{\omega}}\left(\sum_{C_{k l} \in N_{r}(i, j)} \bar{C}_{i j}^{k l} \alpha_{i j}\left\|x_{i j}\right\|_{2}\right. \\
& \left.+\bar{b}_{i j} p_{i j}\left\|\dot{x}_{i j}\right\|_{2}+\left\|I_{i j}\right\|_{2}\right)
\end{aligned}
$$




$$
\begin{gathered}
+\sqrt{\omega}\left[\left(\bar{a}_{i j}+\sum_{C_{k l} \in N_{r}(i, j)} \bar{C}_{i j}^{k l} \alpha_{i j}\right)\left\|x_{i j}\right\|_{2}\right. \\
\left.+\bar{b}_{i j} p_{i j}\left\|\dot{x}_{i j}\right\|_{2}+\left\|I_{i j}\right\|_{2}\right] \\
\leq \frac{1}{\underline{a}_{i j} \sqrt{\omega}}\left(\begin{array}{c}
\sum_{C_{k l} \in N_{r}(i, j)} \bar{C}_{i j}^{k l} \alpha_{i j} h_{i j}^{+} \\
+\bar{b}_{i j} p_{i j} g_{i j}^{+}+\left\|I_{i j}\right\|_{2}
\end{array}\right) \\
\left.+\sqrt{\bar{a}_{i j}+\sum_{C_{k l} \in N_{r}(i, j)} \bar{C}_{i j}^{k l} \alpha_{i j}}\right) h_{i j}^{+} \\
\left.+\bar{b}_{i j} p_{i j} g_{i j}^{+}+\left\|I_{i j}\right\|_{2}\right] \triangleq M_{i j}^{(1)} .
\end{gathered}
$$

Similarly,

$$
\begin{gathered}
x_{i j}\left(\underline{t}_{i j}\right) \geq-\left[\frac{1}{\underline{a}_{i j} \omega} \int_{0}^{\omega}\left|a_{i j}(t) x_{i j}(t)\right| d t+\int_{0}^{\omega}\left|\dot{x}_{i j}(t)\right| d t\right] \\
\geq-\left\{\frac { 1 } { \underline { a } _ { i j } \sqrt { \omega } } \left(\sum_{C_{k l} \in N_{r}(i, j)} \bar{C}_{i j}^{k l} \alpha_{i j} h_{i j}^{+}\right.\right. \\
+\sqrt{\omega}\left[\left(\begin{array}{c}
\bar{b}_{i j} p_{i j} g_{i j}^{+}+\left\|I_{i j}\right\|_{2}
\end{array}\right)\right. \\
\left.\sum_{C_{k l} \in N_{r}(i, j)} \bar{C}_{i j}^{k l} \alpha_{i j}\right) h_{i j}^{+} \\
\left.\left.+\bar{b}_{i j} p_{i j} g_{i j}^{+}+\left\|I_{i j}\right\|_{2}\right]\right\}=-M_{i j}^{(1)} .
\end{gathered}
$$

Thus, for all $i \in \Omega_{1}, j \in \Omega_{2},\left|x_{i j}(t)\right| \leq M_{i j}^{(1)}$, which yields that $\left|x_{i j}\right|_{0} \leq M_{i j}^{(1)}$. Again from (14), we get

$$
\begin{aligned}
\left|\dot{x}_{i j}\right|_{0} & \leq \bar{a}_{i j}\left|x_{i j}\right|_{0}+\sum_{C_{k l} \in N_{r}(i, j)} \bar{C}_{i j}^{k l} \alpha_{i j}\left|x_{i j}\right|_{0}+\bar{b}_{i j}\left|\dot{x}_{i j}\right|_{0}+\bar{I}_{i j} \\
& \leq\left(\bar{a}_{i j}+\sum_{C_{k l} \in N_{r}(i, j)} \bar{C}_{i j}^{k l} \alpha_{i j}\right) M_{i j}^{(1)}+\bar{b}_{i j}\left|\dot{x}_{i j}\right|_{0}+\bar{I}_{i j},
\end{aligned}
$$

which gives $\left|\dot{x}_{i j}\right|_{0} \leq\left(\left(\bar{a}_{i j}+\sum_{C_{k l} \in N_{r}(i, j)} \bar{C}_{i j}^{k l} \alpha_{i j}\right) M_{i j}^{(1)}+\bar{I}_{i j}\right) /(1-$ $\left.\bar{b}_{i j}\right) \triangleq M_{i j}^{(2)}$.

Denote $M^{(1)}=\sum_{i=1}^{m} \sum_{j=1}^{n} M_{i j}^{(1)}, M^{(2)}=\sum_{i=1}^{m} \sum_{j=1}^{n} M_{i j}^{(2)}$, $M^{(3)}=\max _{i \in \Omega_{1}, j \in \Omega_{2}}\left\{\left|\widetilde{I}_{i j}\right| /\left(\widetilde{a}_{i j}-\sum_{C_{k l} \in N_{r}(i, j)} \widetilde{C}_{i j}^{k l} \alpha_{i j}\right)\right\}$. Let $M>$ $\max \left\{M^{(1)}, M^{(2)}, M^{(3)}\right\}, \Omega=\left\{x \in C_{\omega}^{1}:\|x\|_{1}<M\right\}$, and define a bounded bilinear form $[\cdot, \cdot]$ on $C_{\omega} \times C_{\omega}^{1}$ by $[y, x]=$ $\int_{0}^{\omega} y(t) x(t) d t$. Also we define $Q: y \rightarrow$ Coker $(L)$ by $y \rightarrow$ $\int_{0}^{\omega} y(t) d t$. Obviously, $\{x \mid x \in \operatorname{Ker} L \cap \partial \Omega\}=\{x \mid x \equiv M$ or $x \equiv-M\}$. Without loss of generality, we may assume that $x \equiv M$. Let $P_{i j}=-\widetilde{a}_{i j} M-\sum_{C_{k l} \in N_{r}(i, j)} \widetilde{C}_{i j}^{k l} f_{i j}(M) M+\widetilde{I}_{i j}, Q_{i j}=$ $\widetilde{a}_{i j} M+\sum_{C_{k l} \in N_{r}(i, j)} \widetilde{C}_{i j}^{k l} f_{i j}(-M) M+\widetilde{I}_{i j}, i \in \Omega_{1}, j \in \Omega_{2}$. Thus

$$
\begin{aligned}
& {[Q N(x)+Q(r), x][Q N(-x)+Q(r), x]} \\
& =M^{2} \omega^{2} \cdot\left(\begin{array}{c}
P_{11} Q_{11} \\
\vdots \\
P_{m n} Q_{m n}
\end{array}\right) .
\end{aligned}
$$

Considering assumption $\left(\mathrm{H}_{4}\right)$, we know $M>$ $\max _{i \in \Omega_{1}, j \in \Omega_{2}}\left\{\left|\widetilde{I}_{i j}\right| /\left(\widetilde{a}_{i j}-\sum_{C_{k l} \in N_{r}(i, j)} \widetilde{C}_{i j}^{k l} \alpha_{i j}\right)\right\}>0$, and it follows that $\tilde{a}_{i j} M>\sum_{C_{k l} \in N_{r}(i, j)} \widetilde{C}_{i j}^{k l} \alpha_{i j} M+\widetilde{I}_{i j}>$ $\sum_{C_{k l} \in N_{r}(i, j)} \widetilde{C}_{i j}^{k l}\left|f_{i j}(M)\right| M+\widetilde{I}_{i j}$. So we have $\left[Q N\left(x_{i j}\right)+\right.$ $\left.Q\left(r_{i j}\right), x_{i j}\right]\left[Q N\left(-x_{i j}\right)+Q\left(r_{i j}\right), x_{i j}\right]<0$, for all $i \in \Omega_{1}, j \in \Omega_{2}$.

Therefore, by using Lemma 1 , we obtain that (1) has at least one $\omega$-periodic solution. The proof is complete.

\section{Examples}

In this section, we give two examples to demonstrate the results obtained in previous sections.

Consider the following shunting inhibitory cellular neural networks with neutral delays:

$$
\begin{aligned}
\dot{x}_{i j}(t)= & -a_{i j}(t) x_{i j}(t) \\
& -\sum_{C_{k l} \in N_{r}(i, j)} C_{i j}^{k l}(t) f_{i j}\left(x_{k l}\left(t-\tau_{k l}(t)\right)\right) x_{i j}(t) \\
& +b_{i j}(t) \dot{x}_{i j}\left(t-\sigma_{i j}(t)\right)+I_{i j}(t)
\end{aligned}
$$

where $i=1, \ldots, m, j=1, \ldots, n$.

Example 1. Let $m=n=2, f_{i j}(u)=\tanh u=\left(e^{u}-e^{-u}\right) /\left(e^{u}+\right.$ $\left.e^{-u}\right)$. We take

$$
\begin{gathered}
\left(a_{i j}\right)_{3 \times 3}=\left(\begin{array}{cc}
4+0.2 \sin 3 t & 5+0.1 \cos t \\
6+\sin \sqrt{2} t & 5-\cos t
\end{array}\right), \\
\left(C_{i j}\right)_{3 \times 3}=\left(\begin{array}{cc}
0.3-0.1 \sin t & 1+0.2 \cos t \\
0.6|\sin t| & 0.3-0.2 \sin t
\end{array}\right),
\end{gathered}
$$




$$
\begin{aligned}
& \left(b_{i j}\right)_{3 \times 3}=\left(\begin{array}{cc}
0.03 \sin t & 0.1 \\
0.15 & 0.1 \sin 2 t
\end{array}\right), \\
& \left(I_{i j}\right)_{3 \times 3}=\left(\begin{array}{cc}
1.6 & \frac{\cos t+\sin t}{3} \\
\tan \frac{t}{2} & 0.5
\end{array}\right), \\
& \left(\tau_{i j}\right)_{3 \times 3}=\left(\begin{array}{cc}
\sin t \cos t & 0.25 \cos t \\
7 \sin t & \sin 0.1 t
\end{array}\right), \\
& \left(\sigma_{i j}\right)_{3 \times 3}=\left(\begin{array}{cc}
1+\sin 0.5 t & 0.8 \cos t \\
0.3 \sin t & \sin 0.2 t
\end{array}\right) .
\end{aligned}
$$

Obviously, $\left(\mathrm{H}_{1}\right)-\left(\mathrm{H}_{3}\right)$ hold and

$$
\begin{aligned}
\sum_{C_{k l} \in N_{1}(1,1)} \overline{C_{11}^{k l}} & =\sum_{C_{k l} \in N_{1}(1,2)} \overline{C_{12}^{k l}} \\
& =\sum_{C_{k l} \in N_{1}(2,1)} \overline{C_{21}^{k l}} \\
& =\sum_{C_{k l} \in N_{1}(2,2)} \overline{C_{22}^{k l}}=2.7,
\end{aligned}
$$

$p_{11}=1.414, p_{12}=2.236, p_{21}=1.195$, and $p_{22}=1.118$.

Let $\alpha_{i j}=1$, we have $A=\operatorname{diag}(3.8,4.9,5,4)$,

$$
\begin{gathered}
B=\operatorname{diag}(0.042,0.224,0.179,0.112) \\
C=\operatorname{diag}(2.7,2.7,2.7,2.7) \\
D=\operatorname{diag}(0.958,0.776,0.821,0.888) \\
A-C-B D^{-1}(A+C)=\operatorname{diag}(0.815,0.006,0.621,0.455) .
\end{gathered}
$$

It is easy to see that $\left(\mathrm{H}_{4}\right)$ and $\left(\mathrm{H}_{5}\right)$ hold. Therefore, all conditions of Theorem 6 are satisfied, then system (36) has at least one $2 \pi$-periodic solution.

Example 2. Let $m=n=3, f_{i j}(u)=(1 / 2) \sin u$. We take

$$
\begin{gathered}
\left(a_{i j}\right)_{3 \times 3}=\left(\begin{array}{ccc}
4+\sin 3 t & 5+\cos t & 6-\sin t \\
5+\sin 3 t & 6+\sin 2 t & 4+\sin t \\
6+\cos 5 t & 4+\sin t & 5+\cos t
\end{array}\right), \\
\left(C_{i j}\right)_{3 \times 3}=\left(\begin{array}{ccc}
0.1|\cos 3 t| & 0.2|\cos t| & 0.1|\sin t| \\
0.2|\sin 2 t| & 0.3|\sin t| & 0.4|\cos t| \\
0.1|\cos 5 t| & 0.1|\sin t| & 0.2|\sin t|
\end{array}\right), \\
\left(b_{i j}\right)_{3 \times 3}=\left(\begin{array}{ccc}
0.01 \sin t & 0.02 \cos 5 t & 0.01 \sin 2 t \\
0.02 \sin 3 t & 0.03 \sin 2 t & 0.01 \sin 5 t \\
0.03 \cos 3 t & 0.01 \sin t & 0.02 \cos 3 t
\end{array}\right),
\end{gathered}
$$

$$
\begin{aligned}
& \left(I_{i j}\right)_{3 \times 3}=\left(\begin{array}{ccc}
2+\sin t & \cos t & \sin t \\
\sin t & \cos t & 1+\cos t \\
\cos t & \sin 2 t & \sin t+\cos t
\end{array}\right), \\
& \left(\tau_{i j}\right)_{3 \times 3}=\left(\begin{array}{ccc}
0.1 \sin t & 0.5 \cos t & 0.6 \sin t \\
0.8 \sin t & 0.1 \sin t & 0.4 \sin t \\
0.5 \cos t & 0.15 \sin t & 0.55 \cos t
\end{array}\right), \\
& \left(\sigma_{i j}\right)_{3 \times 3}=\left(\begin{array}{ccc}
0.6 \cos t & 0.2 \cos t & 0.15 \sin t \\
0.2 \sin t & 0.75 \sin t & 0.4 \cos 2 t \\
0.8 \cos t & 0.9 \sin t & 0.75 \sin t
\end{array}\right) .
\end{aligned}
$$

Obviously, $\left(\mathrm{H}_{1}\right)-\left(\mathrm{H}_{3}\right)$ hold and

$$
\begin{gathered}
\sum_{C_{k l} \in N_{1}(1,1)} \overline{C_{11}^{k l}}=0.8, \quad \sum_{C_{k l} \in N_{1}(1,2)} \overline{C_{12}^{k l}}=1.3, \\
\sum_{C_{k l} \in N_{1}(1,3)} \overline{C_{13}^{k l}}=1, \\
\sum_{C_{k l} \in N_{1}(2,1)} \overline{C_{21}^{k l}}=1, \quad \sum_{C_{k l} \in N_{1}(2,2)} \overline{C_{22}^{k l}}=1.7, \\
\sum_{C_{k l} \in N_{1}(3,1)} \overline{C_{31}^{k l}}=0.7, \quad \overline{C_{23}^{k l}}=1.3, \\
\sum_{C_{k l} \in N_{1}(3,3)} \overline{C_{33}^{k l}}=1,
\end{gathered}
$$

$p_{11}=1.581, p_{12}=1.118, p_{13}=1.085, p_{21}=1.118, p_{22}=2$, $p_{23}=2.236, p_{31}=2.236, p_{32}=3.162$, and $p_{33}=2$.

Let $\alpha_{i j}=1 / 2$, we have $A=\operatorname{diag}(3,4,5,4,5,3,5,3,4)$,

$$
B=\operatorname{diag}(0.016,0.022,0.011,0.022,0.06 \text {, }
$$

$0.022,0.067,0.032,0.04)$

$C=\operatorname{diag}(0.4,0.65,0.5,0.5,0.85,0.65,0.35,0.65,0.5)$,

$$
\begin{gathered}
D=\operatorname{diag}(0.984,0.978,0.989,0.978,0.94, \\
0.978,0.933,0.968,0.96),
\end{gathered}
$$

$$
\begin{gathered}
A-C-B D^{-1}(A+C) \\
=\operatorname{diag}(2.545,3.245,4.439,3.399,3.777, \\
2.268,4.266,2.229,3.313) .
\end{gathered}
$$

It is easy to see that $\left(\mathrm{H}_{4}\right)$ and $\left(\mathrm{H}_{5}\right)$ hold. Therefore, all conditions of Theorem 6 are satisfied, then system (36) has at least one $2 \pi$-periodic solution.

\section{Conclusion}

In this paper, a class of neutral type shunting inhibitory cellular neural networks is investigated by means of an abstract 
continuous theorem of $k$-set contractive operator and some analysis technique. Sufficient conditions are derived to ensure the existence of periodic solutions. To the best of our knowledge, the results presented here have not appeared in the related literature. Also, illustrative examples are given to demonstrate the effectiveness of the results obtained.

\section{Acknowledgments}

The author is grateful to the referees for their suggestions on the first draft of the paper. This work was supported by the scientific research fund of Fujian Provincial Education Department (JA11309).

\section{References}

[1] A. Bouzerdoum and R. B. Pinter, "Shunting inhibitory cellular neural networks: derivation and stability analysis," IEEE Transactions on Circuits and Systems I, vol. 40, no. 3, pp. 215-221, 1993.

[2] A. Bouzerdoum and R. B. Pinter, "Analysis and analog implementation of directionally sensitive shunting inhibitory cellular neural networks," in Visual Information Processing: From Neurons to Chips, vol. 1473 of Proceedings of SPIE, pp. 29-38, 1991.

[3] Y. K. Li, C. Liu, and L. Zhu, "Global exponential stability of periodic solution of shunting inhibitory CNNs with delays," Physics Letters A, vol. 337, pp. 46-54, 2005.

[4] Y. Xia, J. Cao, and Z. Huang, "Existence and exponential stability of almost periodic solution for shunting inhibitory cellular neural networks with impulses," Chaos, Solitons \& Fractals, vol. 34, no. 5, pp. 1599-1607, 2007.

[5] K. Gopalsamy, Stability and Oscillations in Delay Differential Equations of Population Dynamics, vol. 74, Kluwer Academic, Boston, Mass, USA, 1992.

[6] J. Hale and S. M. Verduyn Lunel, Introduction To Functional Differential Equations, Springer, New York, NY, USA, 1993.

[7] J. H. Park, O. M. Kwon, and S. M. Lee, "State estimation for neural networks of neutral-type with interval time-varying delays," Applied Mathematics and Computation, vol. 203, no. 1, pp. 217-223, 2008.

[8] J. H. Park, O. M. Kwon, and S. M. Lee, "LMI optimization approach on stability for delayed neural networks of neutraltype," Applied Mathematics and Computation, vol. 196, no. 1, pp. 236-244, 2008.

[9] J. H. Park, C. H. Park, O. M. Kwon, and S. M. Lee, "A new stability criterion for bidirectional associative memory neural networks of neutral-type," Applied Mathematics and Computation, vol. 199, no. 2, pp. 716-722, 2008.

[10] S. M. Lee, O. M. Kwon, and J. H. Park, "A novel delay-dependent criterion for delayed neural networks of neutral type," Physics Letters A, vol. 374, no. 17-18, pp. 1843-1848, 2010.

[11] S. Mandal and N. C. Majee, "Existence of periodic solutions for a class of Cohen-Grossberg type neural networks with neutral delays," Neurocomputing, vol. 74, pp. 1000-1007, 2011.

[12] K. Wang and Y. Zhu, "Stability of almost periodic solution for a generalized neutral-type neural networks with delays," Neurocomputing, vol. 73, pp. 3300-3307, 2010.

[13] Y. Li, L. Zhao, and X. Chen, "Existence of periodic solutions for neutral type cellular neural networks with delays," Applied Mathematical Modelling, vol. 36, no. 3, pp. 1173-1183, 2012.
[14] Z. Gui, W. Ge, and X. Yang, "Periodic oscillation for a Hopfield neural networks with neutral delays," Physics Letters A, vol. 364, pp. 267-273, 2007.

[15] K. Deimling, Nonlinear Functional Analysis, Springer, Berlin, Germany, 1985.

[16] W. Petryshynand and Z. Yu, "Existence theorem for periodic solutions of higher order nonlinear periodic boundary value problems," Nonlinear Analysis, vol. 69, pp. 943-969, 1982.

[17] Z. Liu and Y. Mao, "Existence theorem for periodic solutions of higher order nonlinear differential equations," Journal of Mathematical Analysis and Applications, vol. 216, no. 2, pp. 481490, 1997.

[18] S. Lu and W. Ge, "Existence of positive periodic solutions for neutral logarithmic population model with multiple delays," Journal of Computational and Applied Mathematics, vol. 166, no. 2, pp. 371-383, 2004. 


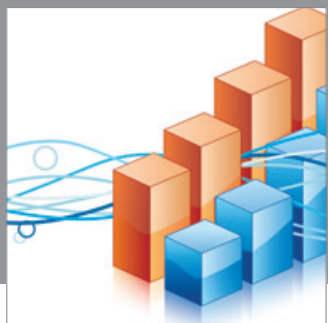

Advances in

Operations Research

mansans

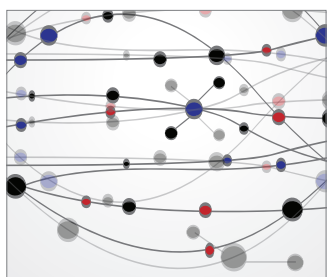

The Scientific World Journal
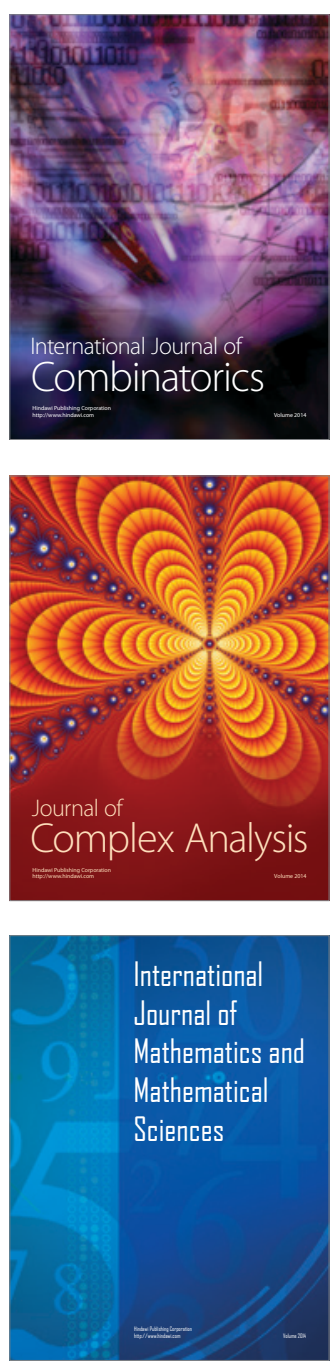
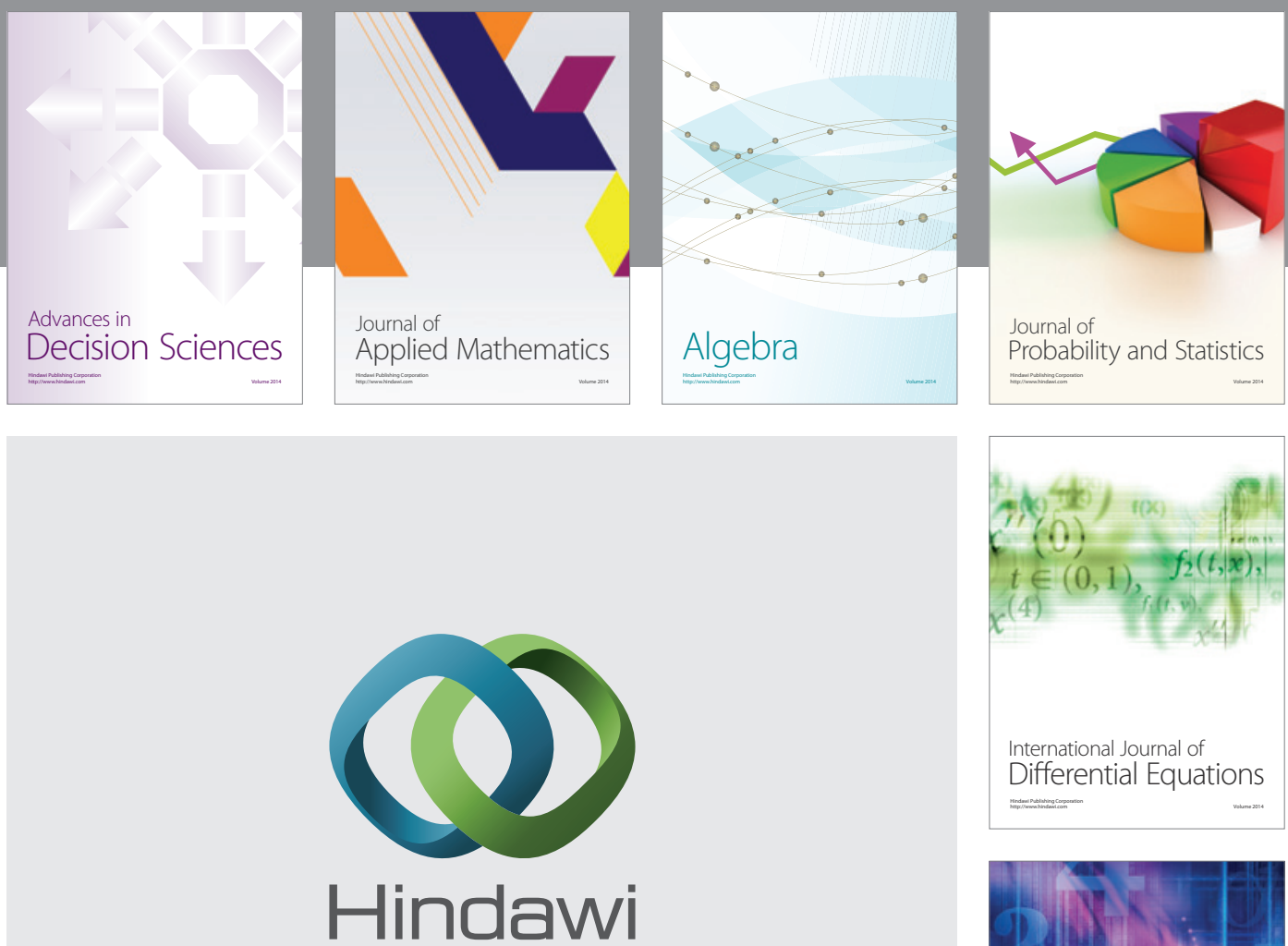

Submit your manuscripts at http://www.hindawi.com
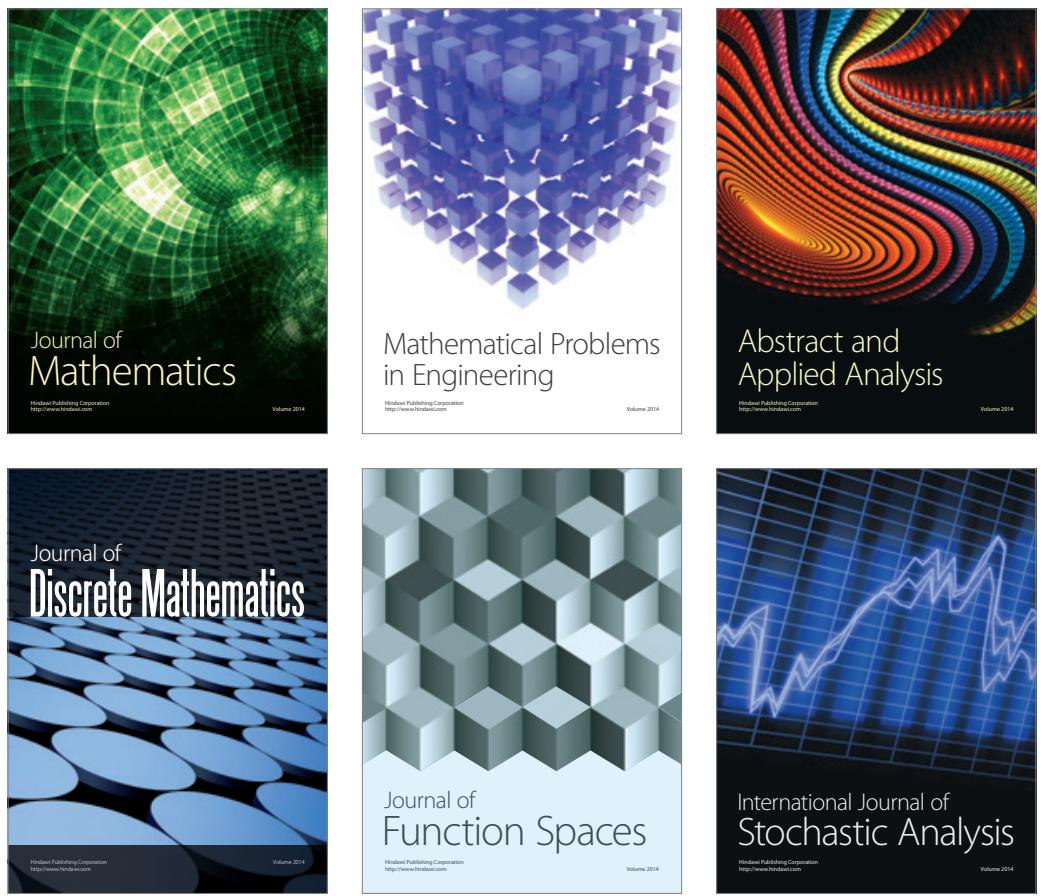

Journal of

Function Spaces

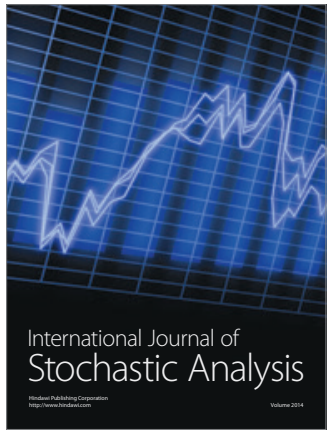

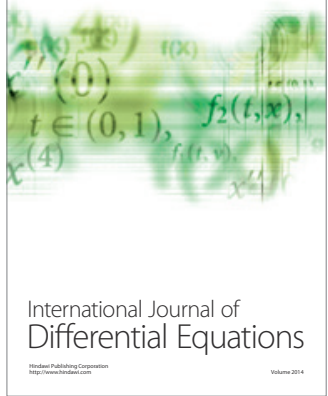
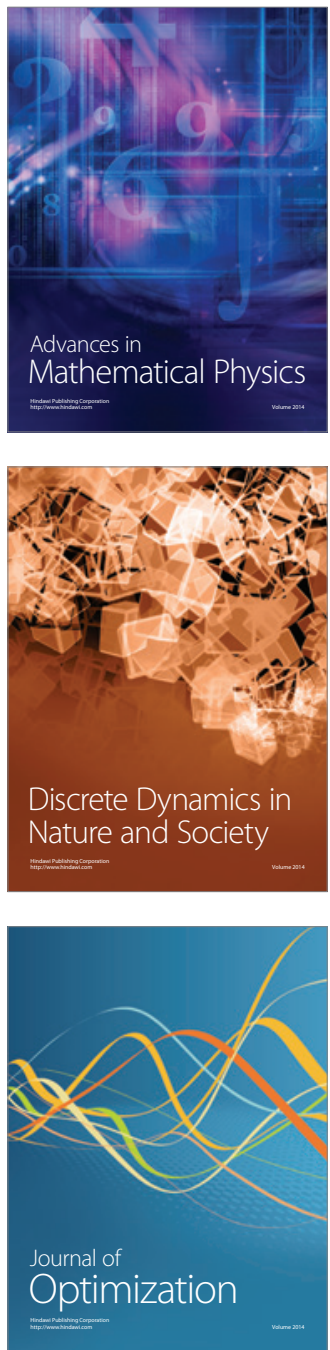